\title{
Effectiveness of universal programmes for the prevention of suicidal ideation, behaviour and mental ill health in medical students: a systematic review and meta-analysis
}

\author{
Katrina Witt, ${ }^{\oplus 1,2}$ Alexandra Boland, ${ }^{2}$ Michelle Lamblin, ${ }^{2}$ Patrick D McGorry, ${ }^{2,3}$ \\ Benjamin Veness, ${ }^{4}$ Andrea Cipriani, ${ }^{\oplus 5}$ Keith Hawton, ${ }^{5}$ Samuel Harvey, ${ }^{6}$ \\ Helen Christensen, ${ }^{6}$ Jo Robinson ${ }^{2}$
}

Additional material is published online only. To view please visit the journal online (http://dx.doi.org/10.1136/ ebmental-2019-300082).

1 Turning Point, Eastern Health Clinical School, Monash

University, Richmond, Victoria, Australia

${ }^{2}$ Orygen, The National Centre for Excellence in Youth Mental Health, University of Melbourne, Melbourne, Victoria, Australia ${ }^{3}$ Centre for Youth Mental Health, University of Melbourne Melbourne, Victoria, Australia

${ }^{4}$ Alfred Health, Melbourne, Victoria, Australia

${ }^{5}$ Department of Psychiatry, University of Oxford, Oxford, UK ${ }^{6}$ Black Dog Institute, Sydney, New South Wales, Australia

\section{Correspondence to}

Dr Katrina Witt, Turning Point, Eastern Health Clinical School, Monash University, Richmond, VIC 3121, Australia; katrina. witt@monash.edu

Preliminary data relating to this review were presented by $A B$ on 30 November 2018 at the 40th Annual Society for Mental Health Research Conference, Noosa Heads, Queensland, Australia

Received 11 January 2019 Revised 25 February 2019 Accepted 17 January 2019 Published Online First 27 March 2019

Check for updates

(c) Author(s) (or their employer(s)) 2019. No commercial re-use. See rights and permissions. Published by BMJ.

To cite: Witt K, Boland A, Lamblin $\mathrm{M}$, et al. Evid Based Mental Health

2019:22:84-90

\begin{abstract}
Question A growing body of work suggests that medical students may be particularly at risk of mental ill health, suicidal ideation and behaviour, resulting in recent calls to develop interventions to prevent these outcomes. However, few reviews have synthesised the current evidence base regarding the effectiveness of these interventions and provided guidance to improve future intervention efforts.
\end{abstract}

Study selection and analysis The authors conducted a systematic review to identify studies of any design reporting the effectiveness of any universal intervention to address these outcomes in medical students. Embase, MEDLINE and PsycINFO databases were searched from their respective start dates until 1 December 2017. Findings Data from 39 studies were included. Most investigated the effectiveness of relatively brief interventions designed to reduce stress; most commonly using mindfulness-based or guided meditation approaches. Only one implemented an intervention specifically designed to address suicidal ideation; none investigated the effectiveness of an intervention specifically designed to address suicidal behaviour. Five investigated the effects of curriculum-level changes. Overall, there was limited evidence of an effect for these programmes at both the postintervention and longest follow-up assessment on depression, anxiety and stress. Conclusions Relatively brief, individually focused, mindfulness-based interventions may be effective in reducing levels of anxiety, depression and stress in medical students in the short term. Effects on suicidal ideation and behaviour, however, remain to be determined. There has been a significant lack of attention on organisational-level stressors associated with medical education and training

\section{BACKGROUND}

Despite suicide being a leading cause of death for those between 15 and 29 years of age globally, ${ }^{1}$ to date, most population-level (or universal) interventions to reduce suicide and related outcomes, including suicidal ideation, and depression in young people have focused on school settings. ${ }^{2}$ While a recent comprehensive review of intervention designed to address suicidal ideation and behaviour in young people recommended psychoeducational
Summary box

What is already known about this subject?

- Higher rates of suicidal ideation and attempted suicide have been reported in medical students as compared with age-matched members of the general population.

- Medical students face a unique range of personal and professional stressors.

- However, medical students are less likely to access services despite better knowledge of appropriate treatment options for mental illness and suicidal ideation and/or behaviour.

What are the new findings?

- Relatively brief mindfulness-based stress management interventions may be effective in reducing anxiety, depression and stress in medical students in the short term.

- Despite recent concerns around suicide rates in both medical students and doctors-intraining, however, we found only one study that evaluated outcomes related to suicidal ideation and none reported outcomes relating to suicidal behaviour.

- Interventions developed to date to address sources of stress in medical students have predominately focused on improving individual resilience and have not addressed the cultural, systemic and structural factors that may also impact on student well-being.

How might it impact on clinical practice in the foreseeable future?

- The study designs used to investigate the effects of these interventions have been relatively weak, and likely the interventions tested have been of too low intensity.

- There has also been a significant lack of attention to organisational-level stressors associated with medical education and training.

approaches coupled with screening showed some promise in reducing levels of suicidal ideation and behaviour in predominately school-aged children, ${ }^{3}$ few of these studies were based in tertiary settings, and none targeted medical students specifically. 
While lower rates of suicide have been observed in tertiary students compared with their non-tertiary educated peers of the same age, ${ }^{4}$ higher rates of suicidal ideation ${ }^{5}$ and attempted suicide $^{6}$ have been reported in medical students as compared with age-matched members of the general population. In a large meta-analysis, almost one-third (27.2\%) of medical students reported depression or symptoms of depression and around 1 in $10(11.1 \%)$ reported suicidal ideation.?

Increased rates of depression, suicidal ideation and behaviour in medical students may be due to a range of unique stressors, including a demanding study load, ${ }^{6}$ which in turn may reduce available time for self-care, ${ }^{8}$ and frequent exposure to death and dying in patients, including suicide death. ${ }^{9}$ Other commonly reported stressors specific to medical education include fear over making mistakes during, for example, clinical rotations, and fear over making the right decision for patients' care. ${ }^{6}$

The format and delivery of medical curricula has also been identified as a specific source of stress. ${ }^{10}$ Frequent examinations, ${ }^{11}$ lengthy academic curricula, ${ }^{12}$ tiered, rather than pass/fail grading systems, ${ }^{13}$ a mismatch between preferred teaching and learning styles ${ }^{14}$ and lack of preparation for career transitions, in particular between preclinical and clinical training ${ }^{15}$ have all been associated with poorer mental health outcomes in medical students. The structure of medical school curricula therefore offers a significant opportunity for intervention. ${ }^{16}$

Medical students are also less likely to access services despite better knowledge of appropriate treatment options for mental illness and suicidal ideation and/or behaviour, as well as knowledge of how to access these services at times of crisis. ${ }^{17}$ According to the first National Survey of Mental Health in Australian Medical Students, embarrassment, fear over a lack of confidentiality and privacy and the impact of having a record of mental health treatment either on registration, the right to practice and/or career progression are common barriers that discourage medical students from seeking help. ${ }^{6}$

\section{OBJECTIVE}

Taken together, these findings suggest there is a need to develop effective universal programmes that can address sources of mental ill health, suicidal ideation and suicidal behaviour in all medical students irrespective of current levels of distress. While a number of universal interventions have been developed to improve general well-being in medical students, these have predominately focused on improving individual resilience ${ }^{18}$ and have not addressed the cultural, systemic and structural factors that may impact on well-being in this population. Therefore, we have conducted a systematic review and meta-analysis of universal interventions aimed at addressing mental ill health, suicidal ideation and behaviour in medical students.

\section{STUDY SELECTION AND ANALYSIS}

We searched Embase, MEDLINE and PsycINFO until 1 December 2017. Ancestry searches were also conducted to locate relevant studies inadvertently missed by the electronic search. Further detail on the electronic search strategy is available in the appendix.

Studies were eligible for inclusion in this review if: (1) they were a randomised controlled trial (RCT), including cluster RCTs, a non-randomised controlled trial (non-RCT), a historically controlled design or uncontrolled longitudinal design; (2) the effectiveness of a universal intervention to address suicidal ideation, suicidal behaviour, depression, anxiety stress and/or burnout was evaluated; (3) participants were medical students; and (4) they were published in English. Studies were limited to English literature due to resourcing constraints. We define universal interventions as those that are delivered to all students irrespective of risk and/or current levels of psychological distress.

All retrieved articles were double-screened by KW and $\mathrm{AB}$ independently. Disagreements were resolved through consensus discussions with ML, JR and BV.

\section{Data extraction}

$\mathrm{KW}$ and $\mathrm{AB}$ independently extracted data and methodological information. The primary outcome assessed was suicidal ideation. Given the associations between suicidal ideation and behaviour, depression, anxiety, stress and burnout in the medical workforce, we also included the following secondary outcome measures: suicidal behaviour (ie, self-harm, attempted suicide and suicide deaths), depression, anxiety, stress and burnout. As we were interested in the effects of the interventions, we reported effects at postintervention and the longest possible follow-up period (where available) for each outcome, following standard approaches. ${ }^{19}$

\section{Study quality}

$\mathrm{KW}$ and $\mathrm{AB}$ independently assessed the quality of each included study. We used the following tools for the different categories of study design included in the review: (1) the Cochrane Risk of Bias tool for RCTs; (2) the Risk of Bias In Non-randomised Studies of Interventions tool for non-RCTs; and (3) the Newcastle-Ottawa Scale (NOS) for longitudinal studies.

\section{Data synthesis and statistical analysis}

Where possible, we synthesised quantitative data between studies using meta-analysis. For continuous outcome data, we calculated the standardised mean difference (SMD) with corresponding 95\% CIs. For dichotomous outcome measures, we calculated the pooled OR with corresponding 95\% CI. Given likely differences in both foci and effects of individually focused interventions compared with curriculum-based interventions, we did not pool data in any meta-analysis across these two broad approaches. Data synthesis was undertaken in Review Manager (RevMan) for Windows, V.5.3, ${ }^{20}$ by implementing the DerSimonian and Laird random effects model.

\section{FINDINGS}

Overall, 1505 records were identified from the electronic databases, and an additional 116 records were identified through hand searching. After removing duplicates and title screening, 314 full-text articles were retrieved for further screening. Finally, a total of 39 studies were included in the systematic review (see appendix for the study inclusion flow chart, table of methodological characteristics and full references for all included studies).

\section{Characteristics of the included studies}

Of the 39 studies included in the review, over half ( 24 studies; $61.5 \%)$ were conducted in the USA and one-third of studies used an RCT design (13 studies; 33.3\%). Only one study investigated the effectiveness of an intervention specifically designed to address suicidal ideation ${ }^{21}$; none reported information of the effectiveness of these programmes for suicidal behaviour.

Most interventions were relatively brief (ie, between 1 and 16 sessions; mean 4.9) delivered according to mindfulness-based principles (18 studies; 46.1\%); stress management training (5 studies; $12.8 \%$ ); psychoeducation (4 studies; 10.2\%); relaxation 
training (2 studies; 5.1\%); or yoga (2 studies; 5.1\%). Other approaches evaluated in single studies included: telephone counselling; omega-3 fatty acid supplementation; and visual journalling. Five studies (12.8\%) investigated the effectiveness of changing from a didactic lecture-based curriculum format to a problem-based format on reducing stress; however, for one of these, insufficient data on our primary or secondary outcomes were reported to enable inclusion in any meta-analysis. ${ }^{22}$

\section{Characteristics of the included participants}

The 39 studies included a total of 7387 medical students (range: 5-1958) aged between 18 years and 32 years of age. Twenty-five studies included information on the gender of participants; in $21(84.0 \%)$ of these, the majority of participants were women.

\section{Study quality}

Owing to the difficulty in ensuring participants and research personnel were blinded to treatment allocation, all RCTs were rated as at unclear risk of bias for these domains. Similarly, all non-RCTs were downgraded owing to a lack of information on bias in the classification of the intervention being reported. For historically controlled studies, most received between three and five stars on the NOS, suggesting a moderate to high risk of bias, with biases most apparent for comparability of the historical control cohort. Finally, for uncontrolled longitudinal studies, bias was most apparent for the measurement of the outcome measure as all studies relied on self-reported information (see appendix for assessments of study quality for included studies).

\section{Effects of individually focused interventions}

Suicidal ideation

None of the studies included information on the proportion of participants reporting suicidal ideation or on suicidal ideation scores at postintervention. One study included data on suicidal ideation at the longest follow-up point (ie, 1 year). There was some evidence of a moderate reduction in the proportion of participants reporting suicidal ideation by this time point in one historically controlled trial of psychoeducation (OR 0.07, $95 \%$ CI 0.01 to 0.59 ; one study). ${ }^{21}$

\section{Depression}

One uncontrolled longitudinal study of a yoga programme included data on the proportion of participants reporting depression at the postintervention assessment; however, there was no evidence of a significant treatment effect for this programme (OR $0.27,95 \%$ CI 0.05 to 1.36 ; one study). ${ }^{23}$

There was also no apparent effect on depression scores (measured continuously) in six RCTs of predominately mindfulness programmes (SMD $-0.52,95 \% \mathrm{CI}-1.18$ to 0.13 ; six studies; $\mathrm{I}^{2}$ : $93.0 \%$; figure 1). There was also no apparent effect in a single RCT of omega-3 fatty acid supplementation (SMD $0.00,95 \% \mathrm{CI}-0.48$ to 0.48$),{ }^{24}$ or in one non-RCT of guided meditation $^{25}$ at the postintervention assessment (SMD -0.08 , $95 \% \mathrm{CI}-0.32$ to 0.16 ; one study; figure 1$)$. In six uncontrolled longitudinal studies of a diverse range of interventions (including relaxation training, yoga and psychoeducation), there was an apparent effect on depression scores (SMD -0.26 , 95\% CI -0.43 to -0.10 ; six studies; $\mathrm{I}^{2}: 0.0 \%$; figure 1 ); however, this effect was reduced to non-significance following the exclusion of studies in which graduate entry medical students were recruited (SMD $-0.18,95 \% \mathrm{CI}-0.40$ to 0.05 ; two studies; $\left.\mathrm{I}^{2}: 0.0 \%\right)$. The exclusion of such studies made no overall difference to either the magnitude or direction of the effect for all other study designs.

By the longest follow-up assessment (ie, 3 months), there was no evidence of a significant effect of guided meditation on the proportion of participants reporting depression in one non-RCT

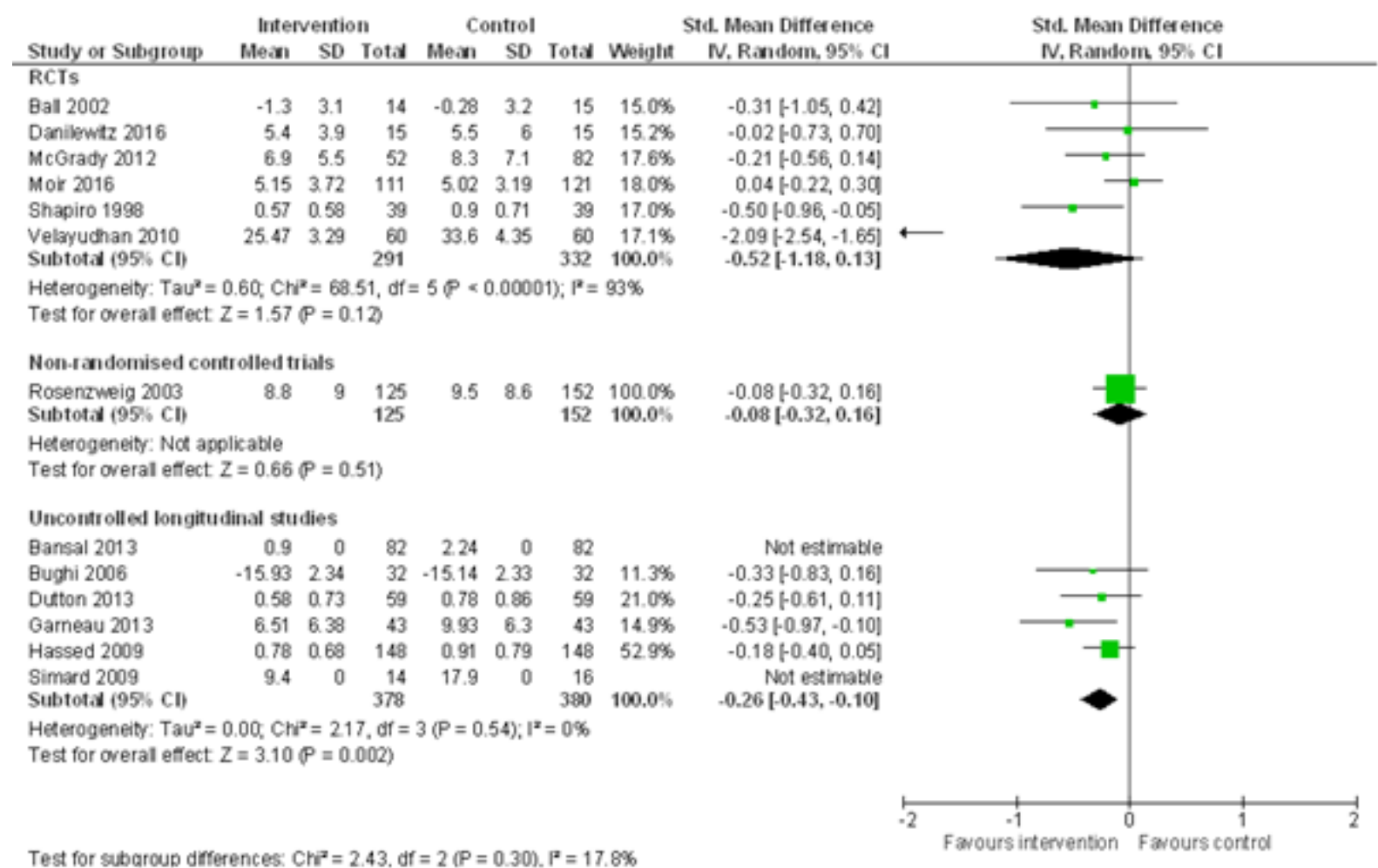

Figure 1 Random effects standardised mean difference (SMD), and accompanying $95 \% \mathrm{Cl}$, on self-reported depression scores at the postintervention assessment. RCTs, randomised controlled trials. 


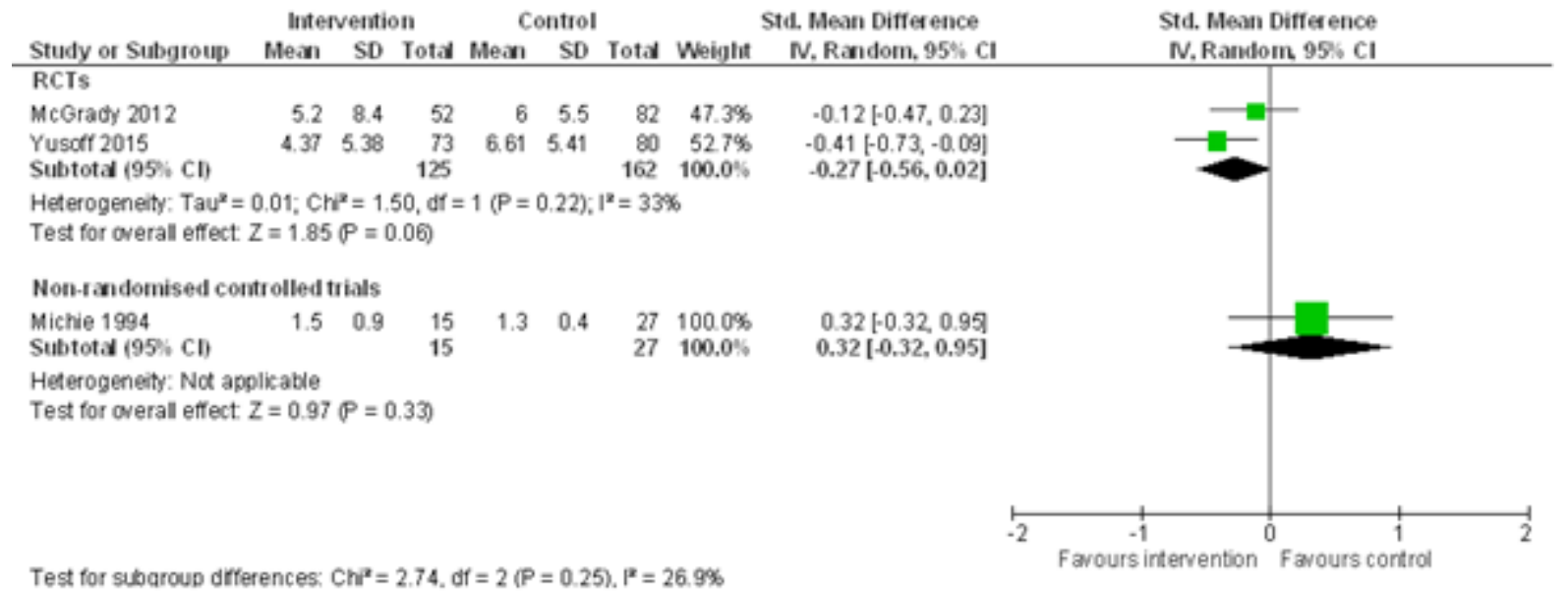

Test for subaroup dfferences: $\mathrm{Ch}^{\mathrm{P}}=2.74, \mathrm{df}=2(\mathrm{P}=0.25), \mathrm{P}^{\mathrm{N}}=26.9 \%$

Figure 2 Random effects standardised mean difference (SMD), and accompanying $95 \% \mathrm{Cl}$, on self-reported depression scores at the longest followup assessment. RCTs, randomised controlled trials.

(OR 1.39, 95\% CI 0.48 to 4.01; one study). ${ }^{26}$ However, there was some evidence of a significant reduction in the proportion reporting depression by this time point in one historically controlled trial of a psychoeducation programme (OR 0.30 ; $95 \%$ CI 0.12 to 0.75 ; one study). ${ }^{21}$ Despite differences in the magnitude of the OR between these studies, there was no evidence of a significant subgroup difference by study design.

There was no significant effect on depression scores at the longest follow-up point for stress management programmes, including mindfulness and psychoeducation approaches in two RCTs (SMD $-0.27,95 \%$ CI -0.56 to 0. 02; two studies; $\mathrm{I}^{2}$ : $33.0 \%$; figure 2 ), or in one non-RCT of psychoeducation
(SMD 0.32, 95\% CI -0.32 to 0.95 ; one study; figure 2 ). ${ }^{27}$ There was no evidence of a significant subgroup difference by study design.

\section{Anxiety}

No study included data on the proportion of participants reporting anxiety at the postintervention assessment. There was no evidence of a significant treatment effect on end-of-treatment anxiety scores in five RCTs of predominately mindfulness-based interventions (SMD $-0.62,95 \% \mathrm{CI}-1.63$ to 0.38 ; five studies; $\mathrm{I}^{2}$ : 97.0\%; figure 3). There was some evidence of a modest

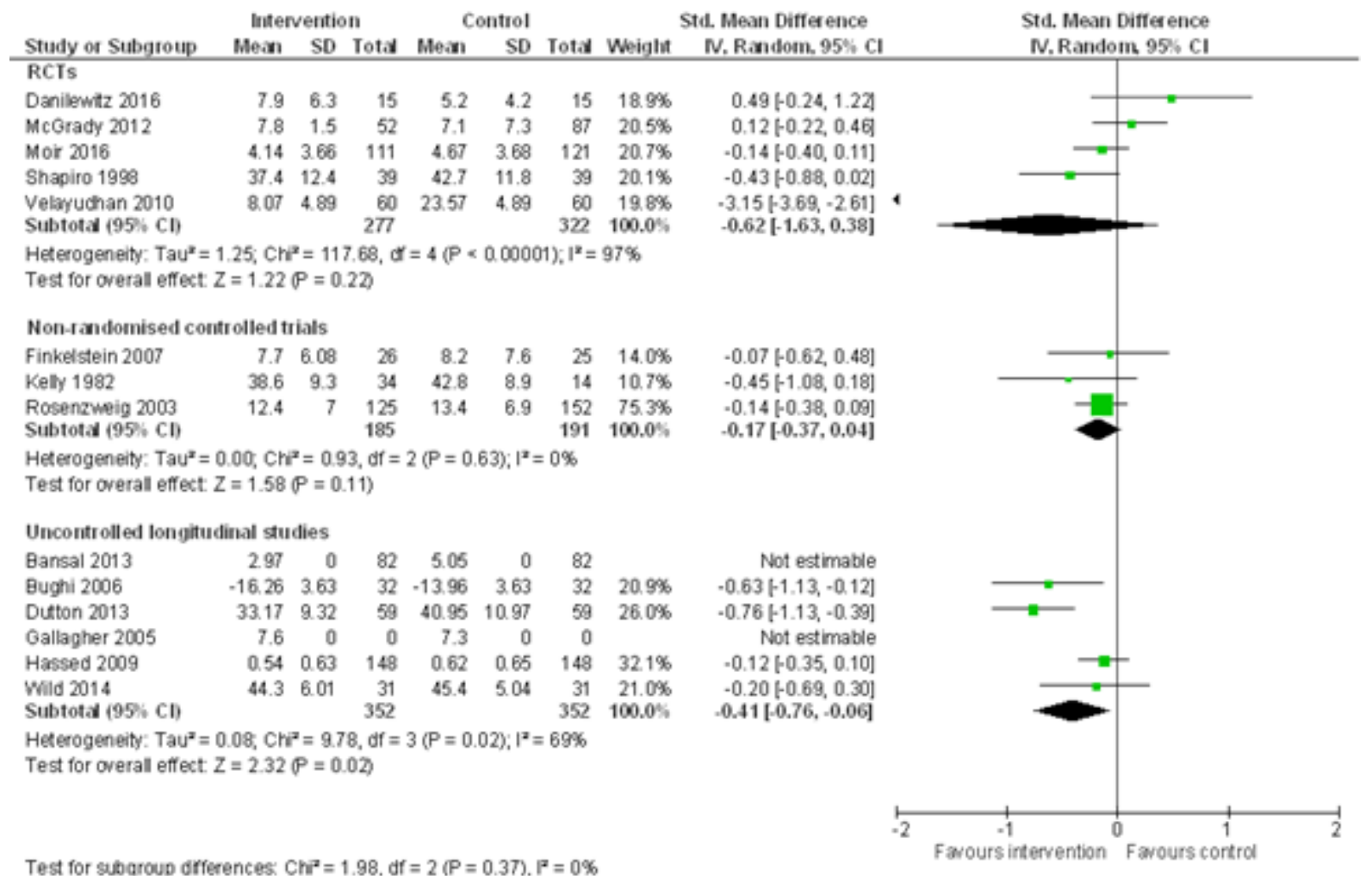

Figure 3 Random effects standardised mean difference (SMD), and accompanying 95\% Cl, on self-reported anxiety scores at the postintervention assessment. RCTs, randomised controlled trials. 


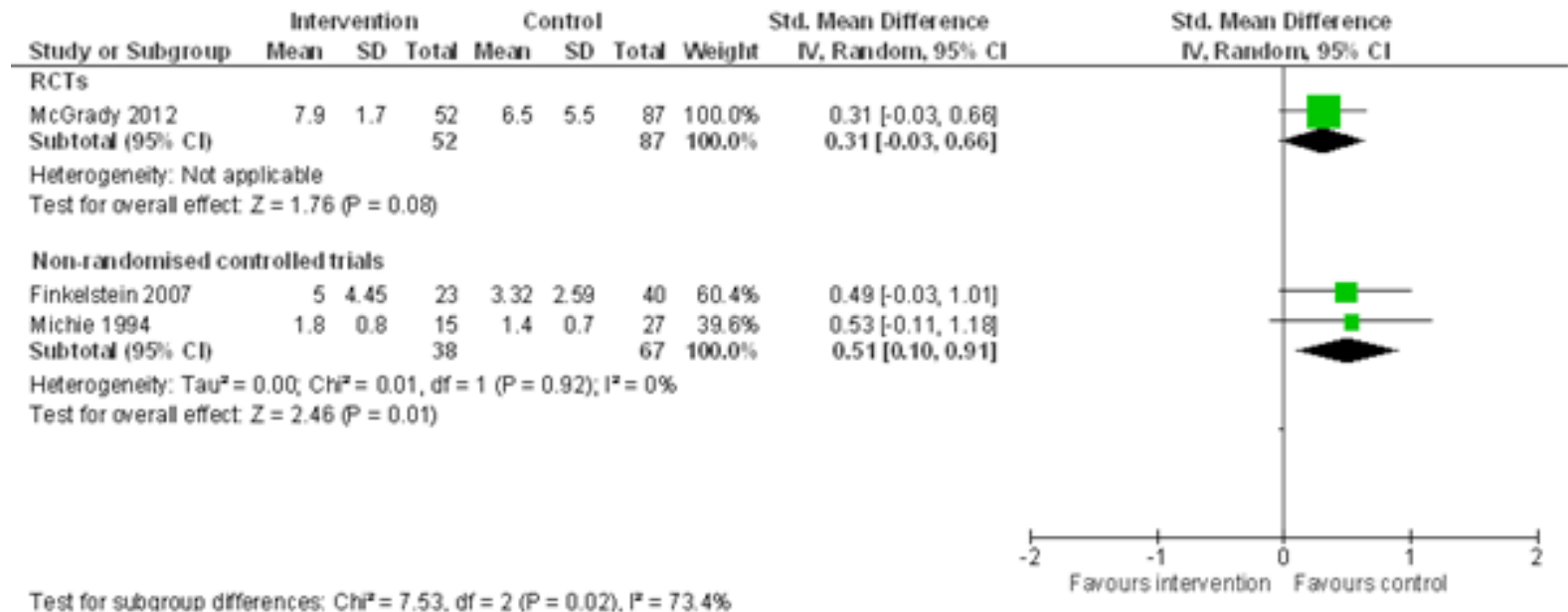

Figure 4 Random effects standard mean difference (SMD), and accompanying $95 \% \mathrm{Cl}$, on self-reported anxiety scores at the final follow-up assessment. RCTs, randomised controlled trials.

treatment effect in favour a single trial of omega-3 essential fatty acid supplementation on anxiety scores at postintervention (SMD $-0.74,95 \% \mathrm{CI}-1.23$ to -0.25$){ }^{24}$

There was no evidence that guided meditation and a groupbased stress management programme may be associated with a reduction in anxiety scores at postintervention based on three non-RCTs (SMD $-0.17,95 \% \mathrm{CI}-0.37$ to 0.04 ; three studies; $\mathrm{I}^{2}: 0.0 \%$; figure 3). However, a positive effect was found in six uncontrolled longitudinal studies that evaluated a number of different approaches designed to reduce anxiety and stress (SMD $-0.41,95 \%$ CI -0.76 to -0.06 ; six studies; $\mathrm{I}^{2}: 69.0 \%$; figure 3). The overall test for subgroup differences by study design was not significant. The effect in favour of stress management programmes on anxiety scores in uncontrolled longitudinal studies was reduced following the exclusion of studies in which graduate-entry medical students were recruited (SMD $-0.12,95 \% \mathrm{CI}-0.35$ to 0.10 ; one study). The exclusion of these studies made no overall difference to either the magnitude or direction of the effect for anxiety scores for all other study designs.

No included study reported data on the proportion of students reporting anxiety at the final follow-up assessment. In terms of anxiety scores at the final follow-up assessment, there was no evidence of a significant treatment effect of a multicomponent stress management programme in one RCT (SMD 0.31, 95\% CI -0.03 to 0.66 ; one study; figure 4$).^{28}$ There was, however, some suggestion of an increase in anxiety scores at final follow-up assessment in two non-RCTs of guided meditation and psychoeducation respectively (SMD $0.51,95 \% \mathrm{CI} 0.10$ to 0.91 ; two studies; $I^{2}: 0.0 \%$; figure 4$)$. The test for subgroup differences by study design was, as a consequence, significant $\chi^{2}=7.53$, $\mathrm{p}=0.02)$.

\section{Stress}

No study included data on the proportion of participants reporting stress at postintervention.

There was no evidence of a significant treatment effect of a mindfulness programme on stress scores at postintervention in one RCT (SMD $-0.32,95 \% \mathrm{CI}-1.04$ to 0.40 ; one study; online supplementary figure 2$),{ }^{29}$ nor for a group-based stress management programme in one non-RCT (SMD -0.38 , $95 \% \mathrm{CI}-1.00$ to 0.25 ; one study; online supplementary figure 2) ${ }^{30}$ However, there was some evidence that these programmes may be associated with a modest reduction in stress in three uncontrolled longitudinal studies (SMD $-0.66,95 \%$ CI -1.32 to -0.00 ; three studies; $\mathrm{I}^{2}: 81.0 \%$; online supplementary figure 2). Despite these differences, there was no evidence of a significant subgroup difference by study design.

No included study reported data on the proportion of students reporting stress at final follow-up. However, two RCTs of mindfulness programmes, ${ }^{31}{ }^{32}$ and one historically controlled trial of a group-based stress management programme, ${ }^{33}$ included information on stress scores at the final follow-up assessment. While mindfulness programmes were associated with a modest effect for stress scores by this time point in two RCTs (SMD -0.28 , $95 \% \mathrm{CI}-0.49$ to -0.07 ; two studies; $\mathrm{I}^{2}: 0.0 \%$; online supplementary figure 3), there was no similar effect found in one historically controlled trial of a group-based psychoeducation programme (SMD $-0.03,95 \% \mathrm{CI}-0.38$ to 0.32 ; one study; online supplementary figure 3 ).

\section{Burnout}

No included study reported data either on the proportions of participants reporting burnout or on burnout scores at the postintervention assessment point.

No study included data on the proportion of students reporting burnout at the final follow-up assessment. One RCT did, however, include information on the effectiveness of a mindfulness programme on burnout scores, with no evidence of a significant effect for this programme by the longest follow-up assessment (SMD $-0.13,95 \% \mathrm{CI}-0.36$ to 0.10 ; one study). ${ }^{31}$

\section{Effects of curriculum-based interventions \\ Suicidal ideation}

No study of a curriculum-based intervention reported data on suicidal ideation at either the postintervention or longest follow-up points.

\section{Depression}

There was no evidence of a treatment effect in favour of curriculum-based interventions on the proportion reporting symptoms of depression by the postintervention assessment in a single study (OR $0.11,95 \%$ CI 0.01 to 1.79 ; one study) ${ }^{34}$ or by the longest follow-up assessment in another single study (OR 0.80, $95 \%$ CI 0.47 to 1.38 ; one study). ${ }^{35}$ 
There was no evidence of a significant reduction in depression scores in one study of a curriculum-based intervention by the longest follow-up assessment (SMD $-0.16,95 \%$ CI -0.38 to 0.06 ; one study). ${ }^{35}$

\section{Anxiety}

No study of curriculum-based interventions reported data on anxiety at the postintervention assessment. By the longest follow-up assessment, there was no evidence of a reduction in anxiety scores in a single study (SMD $-0.02,95 \% \mathrm{CI}-0.17$ to 0.14 ; one study). ${ }^{36}$

\section{Stress}

No study of a curriculum-based intervention reported data on stress at either the postintervention or longest follow-up points.

\section{Burnout}

No study of a curriculum-based intervention reported data on burnout at either the postintervention or longest follow-up points.

\section{CONCLUSIONS AND CLINICAL IMPLICATIONS Key findings}

This review included data from 39 individual studies. Despite recent concerns around suicide rates in both medical students and doctors-in-training, we found only one study that evaluated outcomes related to suicidal ideation, and none reported outcomes relating to suicidal behaviour. Given that so few included studies investigated effects of these interventions on related risk factors, including levels of anxiety, depression and stress, at present there is limited evidence that relatively brief, individually focused mindfulness-based interventions may be effective in reducing these symptoms in medical students.

Most studies investigated the effectiveness of relatively brief programmes for the management of stress, typically between 1 and 16 sessions (mean: 4.9 sessions), delivered over a short time period (mean: 8 weeks). Most interventions were offered during the preclinical years; however, some evidence suggests that levels of suicidal ideation, ${ }^{37}$ depression ${ }^{38}$ and stress ${ }^{39}$ increase throughout the course of the medical degree, peaking in the clinical years of training. ${ }^{40}$ Additionally, stressors likely differ between students in their preclinical years as opposed to those in their clinical training years.

Only four studies investigated the effects of curriculum-based interventions on mental health outcomes for medical students, despite students frequently identifying the structure and format of curricula as a particular source of stress. ${ }^{10}$ Additionally, no study of a curriculum change intervention investigated effects on suicidal ideation or behaviour; however, it should be noted that a number of curriculum-based interventions were excluded from the present review as they did not report data on any of the outcomes of interest. These interventions are therefore worth further investigation.

\section{Limitations of the present review}

Most included studies were conducted in the USA, where the structure of medical education is different to that in many other countries, although similar levels of anxiety, depression and stress have been noted in graduate-entry medical students as compared with their school-leaving counterparts. ${ }^{41}$ We did investigate the effect of including studies in which students were enrolled in a graduate-entry as opposed to an undergraduate medical degree and found effects in favour of individually focused interventions were reduced following the exclusion of studies that predominately recruited postgraduate medical students.

This review aimed to investigate the full spectrum of interventions for the prevention of suicidal ideation and behaviour in medical students and, as such, many meta-analyses pool data across a variety of different intervention approaches. This may have resulted in the very high levels of heterogeneity for some of the results. However, sensitivity analyses omitting studies characterised by very different intervention approaches from the majority did not significantly reduce heterogeneity for these outcomes, suggesting that factors other than intervention approach may also contribute to the variability in treatment effect for these interventions.

\section{Implications}

The majority of studies reported in this review focused on individual-level, mindfulness-based interventions to improve students' resilience and largely ignored the role of inherent structural and systemic stressors. A recent systematic review and meta-analysis of interventions for the prevention of suicide across settings similarly found that most interventions tested in educational settings to date have adopted an individual focus. ${ }^{3}$ However, while mindfulness-based cognitive therapies have demonstrated some evidence of effectiveness in reducing the frequency of selfharming behaviour in young people accessing clinical services, ${ }^{42}$ there is an absence of high-quality evidence at present to suggest this approach may be effective in reducing suicidal ideation and behaviours in non-clinical populations at present. ${ }^{3}$

Medical students are exposed to a number of organisational-level stressors, some of which are relatively embedded in medical training and may be difficult to modify in the short term, while others constitute preventable systems-level failures, such as the fostering of a competitive culture that rewards overwork and environments permissive of intimidation and bullying. ${ }^{43}$ Strategies that address these organisational stressors should therefore be prominent in any intervention aimed at reducing suicidal ideation and behaviour, and mental ill health more generally in this population. ${ }^{44}$ One approach to this might be amending mandatory reporting laws as these may increase stigma and act as a barrier to help-seeking. ${ }^{43}$

\section{CONCLUSIONS}

While brief mindfulness-based stress management interventions may be effective in reducing levels of anxiety, depression and stress in medical students in the short-term, long-term effects on these outcomes, and for suicidal ideation and behaviour in particular, remain to be determined. There has been a particular lack of attention paid to the development and evaluation of interventions to reduce suicidal ideation and behaviour in this population in particular, despite concerns over rising rates. The evidence regarding the effectiveness of these interventions at present is weak for two primary reasons. First, few studies have investigated the effects of these interventions, particularly on levels of suicidal ideation and behaviour in this population. Second, the designs used to investigate the effects of these interventions have been relatively weak, and likely the interventions tested have been of too low intensity. There has also been a significant lack of attention to organisational-level stressors associated with medical education and training.

Contributors All authors approved the manuscript.

Funding The study was funded by the Commonwealth of Australia Department of Health and was part funded by the Prevention Hub, Department of Health.

Individual authors also wish to acknowledge the following sources of funding: KW 
is funded by a postdoctoral fellowship awarded by the American Foundation for Suicide Prevention (PDF-0-145-16), HC is funded by APP1155614 and JR is funded by a National Health and Medical Research Council Career Development Fellowship (ID1142348).

Competing interests One of the authors, AC, is editor-in-chief of Evidence-Based Mental Health.

Patient consent for publication Not required.

Ethics approval The data presented in this review were obtained from previously published reports available within the public domain. Specific institutional approval was therefore not required.

Provenance and peer review Not commissioned; externally peer reviewed.

\section{REFERENCES}

1 World Health Organization. Preventing suicide: a global imperative. Geneva, Switzerland: World Health Organization, 2014.

2 De Silva S, Parker A, Purcell R, et al. Mapping the evidence of prevention and intervention studies for suicidal and self-harming behaviors in young people. Crisis 2013;34:223-32.

3 Robinson J, Bailey E, Witt K, et al. What works in youth suicide prevention? A systematic reviw and meta-analysis. eClinical Med 2018.

4 Mortier P, Auerbach RP, Alonso J, et al. Suicidal thoughts and behaviors among college students and same-aged peers: results from the World Health Organization World Mental Health Surveys. Soc Psychiatry Psychiatr Epidemiol 2018;53:279-88.

5 Tyssen R, Vaglum P, Grønvold NT, et al. Suicidal ideation among medical students and young physicians: a nationwide and prospective study of prevalence and predictors. J Affect Disord 2001;64:69-79.

6 beyondblue. National Mental Health Survey of Doctors and Medical Students. Hawthorne, VIC: beyondblue, 2013.

7 Rotenstein LS, Ramos MA, Torre M, et al. Prevalence of depression, depressive symptoms, and suicidal ideation among medical students: a systematic review and meta-analysis. JAMA 2016;316:2214-36.

8 Pereira MA, Barbosa MA. Teaching strategies for coping with stress--the perceptions of medical students. BMC Med Educ 2013;13:50.

9 Fink-Miller EL. Provocative work experiences predict the acquired capability for suicide in physicians. Psychiatry Res 2015;229:143-7.

10 Dyrbye L, Shanafelt T. A narrative review on burnout experienced by medical students and residents. Med Educ 2016;50:132-49.

11 Matheson KM, Barrett T, Landine J, et al. Experiences of psychological distress and sources of stress and support during medical training: a survey of medical students. Acad Psychiatry 2016;40:63-8.

12 Gazzaz ZJ, Baig M, Al Alhendi BSM, et al. Perceived stress, reasons for and sources of stress among medical students at Rabigh Medical College, King Abdulaziz University, Jeddah, Saudi Arabia. BMC Med Educ 2018;18:29.

13 Spring L, Robillard D, Gehlbach L, et al. Impact of pass/fail grading on medical students' well-being and academic outcomes. Med Educ 2011;45:867-77.

14 Botelho FFR, Bergamo I, Oliveira MCde, et al. Stress level assessment of medical school program and correlations between learning styles and teaching methodologies. MedEdPublish 2017;6:22.

15 Radcliffe C, Lester H. Perceived stress during undergraduate medical training: a qualitative study. Med Educ 2003;37:32-8.

16 Williams DE. The future of medical education: flipping the classroom and education technology. Ochsner J 2016;16:14-15.

17 Tjia J, Givens JL, Shea JA. Factors associated with undertreatment of medical student depression. J Am Coll Health 2005;53:219-24.

18 Shapiro SL, Shapiro DE, Schwartz GE. Stress management in medical education: a review of the literature. Acad Med 2000;75:748-59.

19 Deeks J, Higgins J, Altman D. Analysing data and undertaking meta-analyses. In: Higgins J, Green S, eds. Cochrane Handbook of Systematic Reviews of Interventions. London, UK: The Cochrane Collaboration, 2011.
20 The Cochrane Collaboration. Review Manager (RevMan), version 5.3 [program]. 5.3 version: The Nordic Cochrane Centre, 2014.

21 Thompson D, Goebert D, Takeshita J. A program for reducing depressive symptoms and suicidal ideation in medical students. Acad Med 2010;85:1635-9.

22 Moffat KJ, McConnachie A, Ross S, et al. First year medical student stress and coping in a problem-based learning medical curriculum. Med Educ 2004;38:482-91.

23 Simard A-A, Henry M. Impact of a short yoga intervention on medical students' health: A pilot study. Med Teach 2009;31:950-2.

24 Kiecolt-Glaser JK, Belury MA, Andridge R, et al. Omega-3 supplementation lowers inflammation and anxiety in medical students: a randomized controlled trial. Brain Behav Immun 2011;25:1725-34.

25 Rosenzweig S, Reibel DK, Greeson JM, et al. Mindfulness-based stress reduction lowers psychological distress in medical students. Teach Learn Med 2003;15:88-92.

26 Finkelstein C, Brownstein A, Scott C, et al. Anxiety and stress reduction in medical education: an intervention. Med Educ 2007;41:258-64.

27 Michie S, Sandhu S. Stress management for clinical medical students. Med Educ 1994:28:528-33.

28 McGrady A, Brennan J, Lynch D, et al. A wellness program for first year medical students. Appl Psychophysiol Biofeedback 2012;37:253-60.

29 Danilewitz M, Bradwejn J, Koszycki D. A pilot feasibility study of a peer-led mindfulness program for medical students. Can Med Educ J 2016;7:e31-7.

30 Kelly JA, Bradlyn AS, Dubbert PM, et al. Stress management training in medical school. J Med Educ 1982;57:91-9.

31 de Vibe M, Solhaug I, Tyssen R, et al. Mindfulness training for stress management: a randomised controlled study of medical and psychology students. BMC Med Educ 2013;13:107.

32 Phang CK, Mukhtar F, lbrahim N, et al. Effects of a brief mindfulness-based intervention program for stress management among medical students: the Mindful-Gym randomized controlled study. Adv Health Sci Educ Theory Pract 2015;20:1115-34

33 Holm M, Tyssen R, Stordal KI, et al. Self-development groups reduce medical school stress: a controlled intervention study. BMC Med Educ 2010;10:23.

34 Camp DL, Hollingsworth MA, Zaccaro DJ, et al. Does a problem-based learning curriculum affect depression in medical students? Acad Med 1994;69:S25-7.

35 AlFaris EA, Naeem N, Irfan F, et al. Student centered curricular elements are associated with a healthier educational environment and lower depressive symptoms in medical students. BMC Med Educ 2014;14:192.

36 Zuardi A, De Guerra Prota F, D-b CM. Reduction of the anxiety of medical students after curricular reform [Redução da ansiedade de estudantes de medicina após reforma curricular]. Revista Brasileira de Psiqiatria 2008;30:136-8.

37 Schwenk TL, Davis L, Wimsatt LA. Depression, stigma, and suicidal ideation in medical students. JAMA 2010;304:1181-90.

38 Moutinho IL, Maddalena NC, Roland RK, et al. Depression, stress and anxiety in medical students: A cross-sectional comparison between students from different semesters. Rev Assoc Med Bras 2017;63:21-8.

39 Ludwig AB, Burton W, Weingarten J, et al. Depression and stress amongst undergraduate medical students. BMC Med Educ 2015;15:141.

40 Baldassin S, Alves TC, de Andrade AG, et al. The characteristics of depressive symptoms in medical students during medical education and training: a crosssectional study. BMC Med Educ 2008;8:60.

41 Casey D, Thomas S, Hocking DR, et al. Graduate-entry medical students: older and wiser but not less distressed. Australas Psychiatry 2016;24:88-92.

42 Hawton K, Witt K, Taylor Salisbury T, et al. Interventions for self-harm in children and adolescents. Cochrane Database Syst Rev 2015;12:CD012013.

43 Card AJ. Physician burnout: resilience training is only part of the solution. Ann Fam Med 2018;16:267-70.

44 Kötter T, Pohontsch NJ, Voltmer E. Stressors and starting points for health-promoting interventions in medical school from the students' perspective: a qualitative study. Perspect Med Educ 2015;4:128-35. 